\title{
Research on Series-connected Augmented Railgun Inductance Gradient and Its Influencing parameters
}

\author{
WAN Min ${ }^{1, a}$, WANG Zhiheng ${ }^{1, b}$, LI Xiaojiang ${ }^{1, c}$ \\ ${ }^{1}$ The Academy of Equipment, Beijing, 101416, China \\ awanmin08006328@foxmail.com, bwzh5625412@163.com, cftq_lxj@163.com
}

Keywords: Series-connected Augmented; railgun; inductance gradient; Monte-Carlo algorithm; finite element simulation; influencing parameters

Abstract. The inductance gradient is one of the most important indexes to evaluate the performance of the electromagnetic railgun. It has important theoretical and practical value to research the inductance gradient and its influencing parameters. According to the Biot-Savat's law and the principle of virtual work, the expression of the inductance gradient considered the skin effect of current has been given, and by using Monte-Carlo algorithm the numerical solution has been calculated. In order to verify the correctness and accuracy of the model, the finite element simulation software is used to compare with the numerical solution. Based on the model, the influencing parameters of inductance gradient has been analyzed, and the variation of the inductance gradient has been summarized. The results show that, the inductance gradient decrease with the separation distance between the inside and outside rail, decrease with the height and width of the rail, increase with the displacement of the armature then tend to constant. The research can provide a reference for the design of series-connected augmented railgun.

\section{Introduction}

Series-connected Augmented Railgun (SAG) is a new type of electromagnetic railgun which use several pairs of rails to increase the inductance gradient. Under the same current, SAG's armature can get greater electromagnetic force and higher muzzle velocity [1]. The development of SAG effectively alleviate the contradiction between the rail ablation which caused by high current and the muzzle velocity [2].

The inductance gradient is one of the most important indexes to evaluate the performance of the electromagnetic railgun. It directly affects the velocity and efficiency of the armature [3]. In order to find the relationship between the inductance gradient and the rail structure, geometrical size, material property, and so on, many researchers have carried out the relevant research [4-9].

In this paper, based on Biot-Savat's law and the principle of virtual work, the inductance gradient calculation model of SAG considered current skin effect is established at first. Then by using the calculation model, the influence factors are analyzed, and get the change rule of the influence factors.

\section{Model of the Inductance Gradient}

When the current flows through the rail and armature, a magnetic field is generated between the rails. The current and magnetic field produce the action of the ampere force that push the armature forward through the central axis. The schematic diagram is shown in Fig. 1.

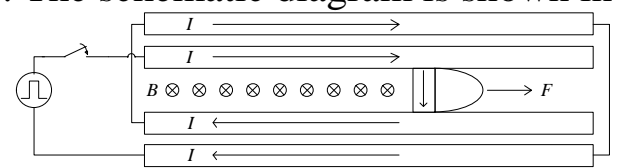

Fig. 1. Schematic diagram of the SAR

The electromagnetic force of the armature can be expressed as

$$
\boldsymbol{F}=\int i \mathrm{~d} \boldsymbol{I} \times \boldsymbol{B}
$$


Where $i \mathrm{~d} \boldsymbol{l}$ is the current vector of the armature, and $\boldsymbol{B}$ is the magnetic induction intensity vector.

And the electromagnetic force of the armature also can be expressed as [10]

$F=\frac{1}{2}\left(L^{\prime}+2 M^{\prime}\right) i^{2}=\frac{1}{2} L_{e}^{\prime} i^{2}$

Where $L$ is the self-inductance of the rail, $M$ is the mutual-inductance of the rail and $L_{e}^{\prime}$ is the equivalent inductance gradient.

From Eq.1 and Eq.2, it can be deduced that

$$
L_{e}^{\prime}=\frac{2 \int i \mathrm{~d} \boldsymbol{l} \times \boldsymbol{B}}{i^{2}}
$$

When a pulse current or an alternating current flow through a conductor, current concentrated on the surface of the conductor, this phenomenon is called skin effect. The depth of skin effect can be expressed as [11]

$$
\delta=\sqrt{\frac{1}{\pi f \mu \sigma}}
$$

Where $\mu$ is the magnetic permeability, $\sigma$ is the electrical conductivity, $f$ is the frequency of the current.

By using the Biot-Savat's law, the magnetic induction intensity $B$ of the position $P$ which generated by direct electric current can be expressed as

$$
B=\mu_{0} I(\cos \alpha+\cos \beta) / 4 \pi r
$$

Where $\mu_{0}$ is the permeability of vacuum, $r$ is the distance from $P$ to the conductor, $\alpha, \beta$ is the angle between $P$ and the conductor.

Consider the skin effect and proximity effect, the following assumptions are proposed:

1) The current is evenly distributed in the depth of skin effect;

2) The top and bottom surface current distribution is uniform, and the internal and external surface current distribution is proportional.

In this setting, the distribution coefficient of inside rail current is $C_{1}, C_{2}$, outside rail current is $C_{3}, C_{4}$, usually $C_{1}>C_{2}, C_{3}<C_{4}$ and $C_{1(3)}+C_{2(4)}=2$.

Based on above condition, the force on the armature that was given in Fig. 2 will be calculated. Assume the current is $I$, the length of the rail and armature are $L$ and $d$.

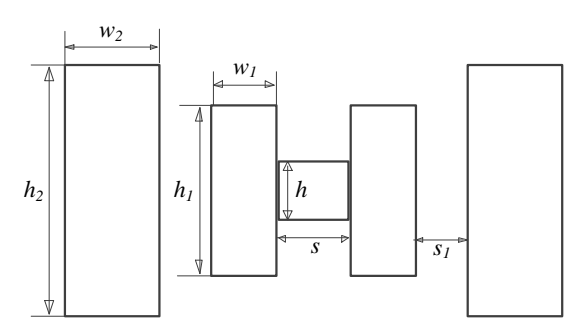

Fig. 2. Cross section of the rail and armature

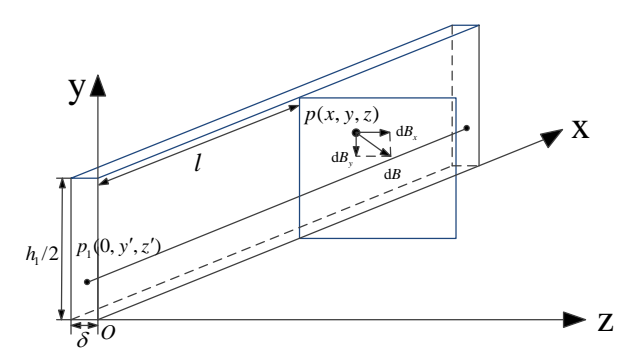

Fig. 3. The magnetic flux density of the internal surface of the inside rail

First, the force generated by the current that distribute on the internal surface of the inside rail should be calculated. The current value can be expressed as $I_{1}=\frac{C_{1} h_{1}}{2\left(h_{1}+w_{1}\right)} I$, so in position $p(x, y, z)$ shown in Fig. 3, the magnetic induction intensity can be expressed as 


$$
\begin{aligned}
\mathrm{d} B & =\frac{\mu_{0} \mathrm{~d} I}{4 \pi r}(\cos \alpha+\cos \beta) \\
& =\frac{\mu_{0} C_{1} I}{8 \pi \delta\left(h_{1}+w_{1}\right)} \frac{1}{\sqrt{\left(y-y^{\prime}\right)^{2}+\left(z-z^{\prime}\right)^{2}}}\left(\frac{x}{\sqrt{x^{2}+\left(y-y^{\prime}\right)^{2}+\left(z-z^{\prime}\right)^{2}}}+\right. \\
& \left.\frac{l-x}{\sqrt{(l-x)^{2}+\left(y-y^{\prime}\right)^{2}+\left(z-z^{\prime}\right)^{2}}}\right) \mathrm{d} y^{\prime} \mathrm{d} z^{\prime}
\end{aligned}
$$

For the convenience of reading, the following replacements are made in the following article:

$\mathrm{A}=\left(y-y^{\prime}\right)^{2}+\left(z-z^{\prime}\right)^{2} \quad, \quad \mathrm{~B}=x^{2}+\left(y-y^{\prime}\right)^{2}+\left(z-z^{\prime}\right)^{2} \quad, \quad \mathrm{C}=(l-x)^{2}+\left(y-y^{\prime}\right)^{2}+\left(z-z^{\prime}\right)^{2}$, $\mathrm{D}=(L-x)^{2}+\left(y-y^{\prime}\right)^{2}+\left(z-z^{\prime}\right)^{2}$

Because of the symmetry of the rail, the magnetic induction intensity in the $Z$ direction will be offset, so the magnetic induction intensity in the Y direction is expressed as

$$
\mathrm{d} B_{y}=\mathrm{d} B \cos \theta=\frac{\mu_{0} C_{1} I}{8 \pi \delta\left(h_{1}+w_{1}\right)} \frac{z-z^{\prime}}{\mathrm{A}}\left(\frac{x}{\sqrt{\mathrm{B}}}+\frac{l-x}{\sqrt{\mathrm{C}}}\right) \mathrm{d} y^{\prime} \mathrm{d} z^{\prime}
$$

The force of the armature is expressed as

$$
F_{L 1}=\left|\int \mathrm{d} I^{\prime} \mathrm{d} z \times B_{y}\right|=\frac{\mu_{0} C_{1} I^{2}}{8 \pi \delta^{2} h\left(h_{1}+w_{1}\right)} \int_{0}^{s} \int_{-h / 2}^{h / 2} \int_{l}^{l+\delta}\left\{\int_{-\delta}^{0} \int_{-h_{1} / 2}^{h_{1} / 2} \frac{z-z^{\prime}}{\mathrm{A}}\left(\frac{x}{\sqrt{\mathrm{B}}}+\frac{l-x}{\sqrt{\mathrm{C}}}\right) \mathrm{d} y^{\prime} \mathrm{d} z^{\prime}\right\} \mathrm{d} x \mathrm{~d} y \mathrm{~d} z
$$

The total force of the armature that generated by the current that distribute on the internal surface of the inside rail is expressed as

$$
F_{1}=F_{L 1}+F_{R 1}=K_{1} I^{2}
$$

Where $F_{R 1}=F_{L 1}$.

$$
K_{1}=\frac{\mu_{0} C_{1}}{4 \pi \delta^{2} h\left(h_{1}+w_{1}\right)} \int_{0}^{s} \int_{-h / 2}^{h / 2} \int_{l}^{l+\delta}\left\{\int_{-\delta}^{0} \int_{-h_{1} / 2}^{h_{1} / 2} \frac{z-z^{\prime}}{\mathrm{A}}\left(\frac{x}{\sqrt{\mathrm{B}}}+\frac{l-x}{\sqrt{\mathrm{C}}}\right) \mathrm{d} y^{\prime} \mathrm{d} z^{\prime}\right\} \mathrm{d} x \mathrm{~d} y \mathrm{~d} z
$$

In the same way, the force of the armature that generated by the current that distribute on other surface can be expressed as

$$
F_{i}=F_{L i}+F_{R i}=K_{i} I^{2}
$$

Where

$$
\begin{aligned}
& K_{2}=\frac{\mu_{0} C_{2}}{4 \pi \delta^{2} h\left(h_{1}+w_{1}\right)} \int_{0}^{s} \int_{-h / 2}^{h / 2} \int_{l}^{l+\delta}\left\{\int_{-w_{1}}^{-w_{1}+\delta} \int_{-h_{1} / 2}^{h_{1} / 2} \frac{z-z^{\prime}}{\mathrm{A}}\left(\frac{x}{\sqrt{\mathrm{B}}}+\frac{l-x}{\sqrt{\mathrm{C}}}\right) \mathrm{d} y^{\prime} \mathrm{d} z^{\prime}\right\} \mathrm{d} x \mathrm{~d} y \mathrm{~d} z \\
& K_{3(4)}=\frac{\mu_{0}}{4 \pi \delta^{2} h\left(h_{1}+w_{1}\right)} \int_{0}^{s} \int_{-h / 2}^{h / 2} \int_{l}^{l+\delta}\left\{\int_{-w_{1}}^{0} \int_{h_{1} / 2-\delta}^{h_{1} / 2} \frac{z-z^{\prime}}{\mathrm{A}}\left(\frac{x}{\sqrt{\mathrm{B}}}+\frac{l-x}{\sqrt{\mathrm{C}}}\right) \mathrm{d} y^{\prime} \mathrm{d} z^{\prime}\right\} \mathrm{d} x \mathrm{~d} y \mathrm{~d} z \\
& K_{5}=\frac{\mu_{0} C_{3}}{4 \pi \delta^{2} h\left(h_{2}+w_{2}\right)} \int_{0}^{s} \int_{-h / 2}^{h / 2} \int_{l}^{l+\delta}\left\{\int_{-\delta-s_{1}-w_{1}}^{-s_{1}-w_{1}} \int_{-h_{1} / 2}^{h_{1} / 2} \frac{z-z^{\prime}}{\mathrm{A}}\left(\frac{x}{\sqrt{\mathrm{B}}}+\frac{L-x}{\sqrt{\mathrm{D}}}\right) \mathrm{d} y^{\prime} \mathrm{d} z^{\prime}\right\} \mathrm{d} x \mathrm{~d} y \mathrm{~d} z \\
& K_{6}=\frac{\mu_{0} C_{4}}{4 \pi \delta^{2} h\left(h_{2}+w_{2}\right)} \int_{0}^{s} \int_{-h / 2}^{h / 2} \int_{l}^{l+\delta}\left\{\int_{-w_{2}-s_{1}-w_{1}}^{\delta-w_{2}-s_{1}-w_{1}} \int_{-h_{1} / 2}^{h_{1} / 2} \frac{z-z^{\prime}}{A}\left(\frac{x}{\sqrt{B}}+\frac{L-x}{\sqrt{D}}\right) \mathrm{d} y^{\prime} \mathrm{d} z^{\prime}\right\} \mathrm{d} x \mathrm{~d} y \mathrm{~d} z \\
& K_{7(8)}=\frac{\mu_{0}}{4 \pi \delta^{2} h\left(h_{2}+w_{2}\right)} \int_{0}^{s} \int_{-h / 2}^{h / 2} \int_{l}^{l+\delta}\left\{\int_{-w_{2}-s_{1}-w_{1}}^{-s_{1}-w_{1}} \int_{h_{1} / 2-\delta}^{h_{1} / 2} \frac{z-z^{\prime}}{A}\left(\frac{x}{B}+\frac{L-x}{\sqrt{D}}\right) \mathrm{d} y^{\prime} \mathrm{d} z^{\prime}\right\} \mathrm{d} x \mathrm{~d} y \mathrm{~d} z
\end{aligned}
$$

In summary, the total force can be expressed as

$$
F=\sum_{i=1}^{8} F_{i}=\sum_{i=1}^{8} K_{i} I^{2}
$$

Substitute Eq. 13 in Eq. 3, the equivalent inductance gradient can be expressed as

$$
L_{e}^{\prime}=2 \sum_{i=1}^{8} K_{i}
$$




\section{Model verification}

In this paper three kinds of SAR with different parameters in Table 1 are designed. To verify the correctness of the model, the finite element method is used to compare with the result calculated by Eq. 14.

Table 1. Design parameters of SAR

\begin{tabular}{cccccccccc}
\hline Parameters/mm & $d$ & $h$ & $S$ & $L$ & $h_{1}$ & $w_{1}$ & $h_{2}$ & $w_{2}$ & $s_{1}$ \\
\hline Type 1 & 20 & 20 & 20 & 1000 & 40 & 10 & 40 & 10 & 10 \\
Type 2 & 30 & 30 & 30 & 1000 & 40 & 15 & 60 & 20 & 10 \\
Type 3 & 20 & 20 & 20 & 800 & 20 & 5 & 40 & 10 & 5 \\
\hline
\end{tabular}

In the finite element method, the inductance gradient at $x$ is expressed as

$L_{e}^{\prime}(x)=\lim _{\Delta x \rightarrow 0} \frac{L_{e}(x+\Delta x)-L_{e}(x-\Delta x)}{2 \Delta x}$

In order to get the approximate value of $L_{e}^{\prime}(x)$, make $\Delta x=50 \mathrm{~mm}$, so Eq. 15 transform to Eq. 16 .

$L_{e}^{\prime}(x)=\frac{[L(x+\Delta x)+2 M(x+\Delta x)]-[L(x-\Delta x)+2 M(x-\Delta x)]}{2 \Delta x}$

The finite element model built in Maxwell 16.0 is given in Fig. 4. In the software, set solution type as Eddy Current, define the rail material as copper and define the armature material as aluminum. Set $I=2 \mathrm{MA}, f=1 \mathrm{kHz}, x=450 \mathrm{~mm}$. Then use the software and Eq. 16 to calculate $L_{e}^{\prime}(x)$.

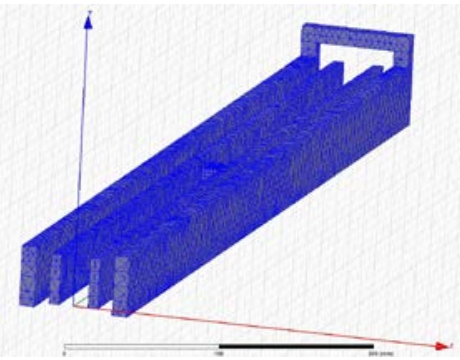

Fig. 4. The three-dimensional finite element model

To Eq. 14, it is a multiple integral summation that is hard to calculate the result. In this paper, the Monte-Carlo algorithm is used to solve the problem in Matlab [12]. According to the simulation results of Maxwell, the distribution coefficient of inside rail current is $C_{1}=1.9 、 C_{2}=0.1$, and the distribution coefficient of outside rail current is $C_{3}=0.4 、 C_{4}=1.6$.

Table 2. Comparison of inductance gradient

\begin{tabular}{cccc}
\hline Parameters $/(\mu \mathrm{H} / \mathrm{m})$ & $L_{e}{ }^{\prime}$ by Matlab & $L_{e}{ }^{\prime}$ by Maxwell & deviation \\
\hline Type 1 & 0.722 & 0.742 & $2.70 \%$ \\
Type 2 & 0.844 & 0.888 & $4.95 \%$ \\
Type 3 & 0.968 & 1.014 & $4.54 \%$ \\
\hline
\end{tabular}

Table 2 shows the results of the comparison. And from Table 2 the model established in this paper can be proved to be correct and accurate.

\section{Influencing parameter analysis}

From the mathematical model, it can be seen that the inductance gradient is related to the distance between the inside and outside rail, the size of the rail and the displacement of the armature. Refer to the designed type 1, analysis the variation of inductance gradient with the parameters. 


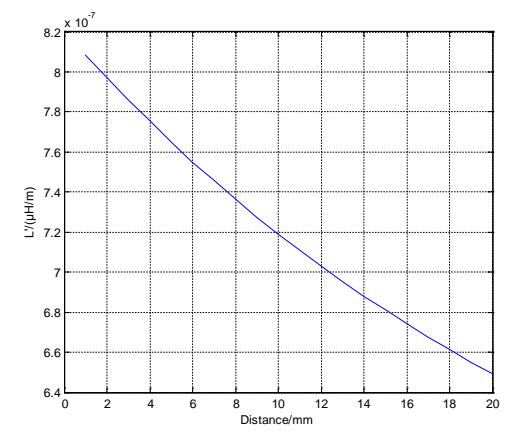

Fig. 5. The curve of the inductance gradient change with the distance between the inside and outside rail

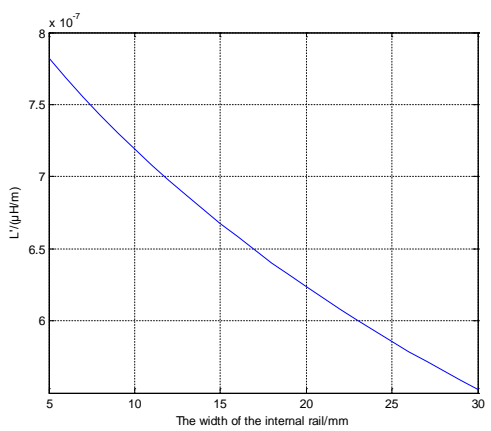

a) Inside rail

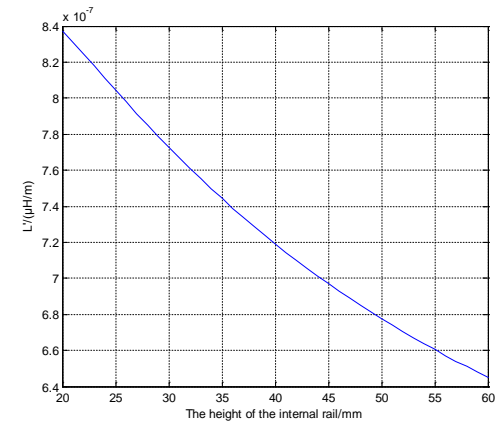

a) Inside rail

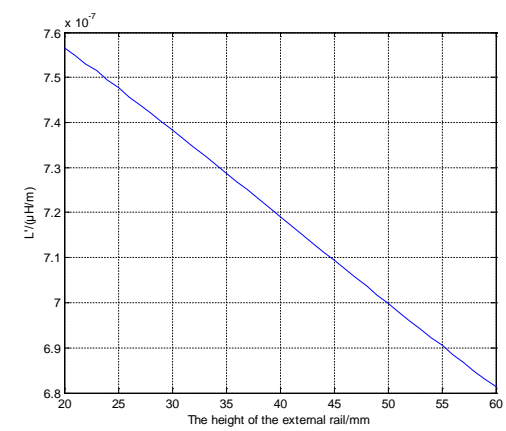

b) Outside rail

Fig. 6. The curve of the inductance gradient change with the height of the rail

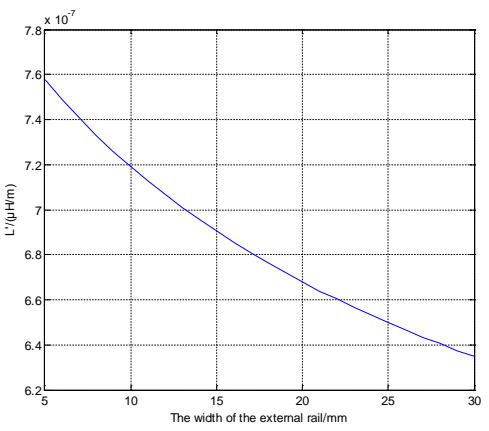

b) Outside rail

Fig. 7. The curve of the inductance gradient change with the width of the rail

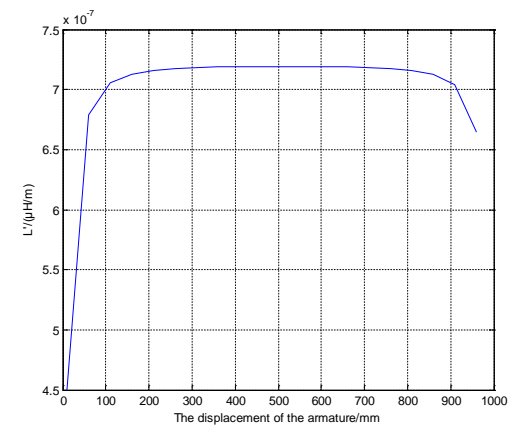

Fig. 8. The curve of the inductance gradient change with the displacement of the armature

The curve of the variation of the inductance gradient along with the distance between the inside and outside rail is shown in Fig. 5. The inductance gradient decrease with the increase of the distance, because when the distance increase, the magnetic induction intensity caused by the outside rail become smaller. In the design of SAR, the distance between the inside and outside rail should reduce as small as possible to enhance the inductance gradient.

The curves of the variation of the inductance gradient along with the height of the rail are shown in Fig. 6. The inductance gradient decrease with the increase of the height, because when the height increase, the section area of the rail becomes lager, and cause the current density to decrease, the increase of the height also cause the distance between the top and bottom surface to increase, all of this makes the magnetic induction intensity become smaller, the inductance gradient also decrease with it. So in the design of SAR, under the premise of ensuring the structural strength, the rail with same height of the armature is better.

The curves of the variation of the inductance gradient along with the width of the rail are shown in Fig. 7. The inductance gradient decrease with the increase of the width. Like the change of the height, the current density decrease and distance between the internal and external surface increase, that makes the magnetic induction intensity become smaller and the inductance gradient decrease. So in the design of SAR, under the premise of ensuring the structural strength, thinner rail should be first considered.

The curve of the variation of the inductance gradient along with the displacement of the armature is shown in Fig. 8. The inductance gradient increase with the increase of the displacement of the armature at first, then tend to a certain value. This result is in accordance with the "four times diameter rule" in literature [11]. When the armature start to move, the electric rail is too short to produce a large magnetic field, but when the armature move forward to the "four times diameter", the magnetic induction intensity tend to maximum, and the inductance gradient tend to a certain value. In 
launching experiments, the armature should be placed in the "four times diameter" to ensure the maximum force at the beginning.

\section{Summary}

Based on Biot-Savat's law and the principle of virtual work, the inductance gradient calculation model of SAG has been built in this paper. And by using the model the variation of inductance gradient with the parameters have been analyzed. The analysis results propose several design suggestion. Under the premise of ensuring the structural strength, to enhance the inductance gradient the distance between the inside and outside rail should reduce as small as possible, the height and width of the rail may take a smaller values, and the armature should be placed in the "four times diameter" to ensure the maximum force at the beginning in a launching experiment.

\section{References}

[1] Y. Wang and F. Xiao: The principle of electrical gun (National Defence Industry Press, China 1995).

[2] J. Gallant: Parametric study of an augmented railgun. IEEE Transactions on Magnetics, vol. 39 no. 1(2003), p. 451

[3] A. Keshtkar: Effect of rail dimension on current distribution and inductance gradient. IEEE Transactions on Magnetics, vol. 41 no. 1(2005), p. 383

[4] J. X. Nie, J. J. Han, Q. J. Jiao, et al: An Analytic Expression of Inductance Gradient for Rail-Type Electromagnetic Launcher. IEEE Transactions on Plasma Science, vol. 39 no. 3(2011), p. 931

[5] S. Bayati, K. Asghar and A. Makki: Magnetic Field and Inductance Gradient at the Circular Railgun. 16th International Symposium on Electromagnetic Launch Technology Proceedings, (2012)

[6] A. Keshtkar and S. Bayati: Effect of rail's material on railgun inductance gradient and losses. 14th International Symposium on Electromagnetic Launch Technology Proceedings, (2008)

[7] D. Keefer, R. Crawford and J. Taylor: Inductance Gradient Scaling Experiments in an Augmented Railgun. IEEE Transactions on Magnetics, vol. 31 no. 1(1995), p. 326

[8] S. B. Liu, J. J. Ruan, Y. D. Zhang, et al: Calculation of Rail Gun Inductance Gradient by Finite Element Method. Electrical Engineering, (2008), p. 132

[9] R. Xu , W. Q. Yuan, W. P. Cheng, et al: Simulation and Analysis of Electromagnetic Field for Augmented Railgun. High Voltage Engineering, vol. 4 no. 40(2014), p. 1065

[10] Y. J. Zhang, M. Y. Sun and P. L. Guo: Comparison of performances between stack and plane turn-augmented railgun. IEEE Conference Publications, (2011), p. 121

[11]R A Marshall and Wang Y: Railguns:their science and technology (Machine Press, China 2004).

[12]M. H. Ren, L. F. Liu and H. F. Mei: Editing program with Monte-Carlo method for calculating multiple integral. Journal of Hunan University of Arts and Science(Natural Science Edition), vol. 23 no. 4(2011), p. 1 